\title{
Prueba de la Sulfofenolptaleina en el embarazo normal
}

\author{
Por el doctor Jaime Corral Madonado, \\ Ex-Jefe de Clinica Obstetrica de la Universidad Nacional
}

Hemos considerado como normales todas aquellas enfermas en las cuales la tensión arterial máxima no ha txcedido de $140 \mathrm{~mm}$. de $\mathrm{Hg}$., y, que en el examen parcial de orina, que siste aticamente pract camos antes de toda prueba, no hayan presentado albúmina o elementos arormales que nos hubieran techo sospechar la insuficiencia renal; además, previo examen clínico de la enferma nos hemos cerciorado de la ausencia de urcmas $u$ otros signos de intoxicacin gravidica, asi como de la concon: tancia con el cmbarazo de otras - Cermedades agudas o cronicas.

La tienica que hemos seguido, y no está por demas resumir bre- nente aqui ha sido la siguiente:

1. Por cateterismo, evacuar la vea y cerciorarse de que ésta queda - mpletamente vacia; en la muestra dt orina asi obtenida practicar el exaI n parcial.

$2^{\circ}$ Hacer ingerir a la enferma 250 c. de agua.

3t Qunce minutes despues de la i. estion de agua, aphenlo por via i) mavenosa $1 \mathrm{cc}$ exacto de solucion de Sulfofenolptaleina que contiene
0.006 gramos de sustancia, $y$ anotar la hora exacta en que ésta operación se ha practicado.

$4^{\circ}$ A partir del momento de la inyección, recolectar la orina eliminada cada cuarto de hora y en el total de una ! ra; anotar la hora exacta en que comenzó a eliminarse la sulfofenoiptaleina; $y, \tan$ pronto como haya terminado la prueba, dosificar los porcentajes de sustancia eliminados en cada una de las muestras de orina recogidas.

Para poder anotar la hora exacta en que la Sulfofenolptaleina comienza a eliminarse por la orina, es reguisito indispensable recoger la primema muestra en un recipiente que contenga 5 cc. de una solución de soda cáustica al $50 \%$; el color rojo que la Sulfofenolptalena desarrolla en presen a de las soluciones alealinas, permite facilmente apreciar d momento en que ista comenzó a eliminarse.

Practicada la prucba en la forma anteriormente expuesta, y habenco observido una diferencia notosa on el nodo de elminación de esta sustancia durante la primera y la so 
gunda mitad del embarazs, hemos resumido en dos cuadros distintos las observaciones de las enfermas correspondientes a cada uno de estos grupos

\section{PROMEDIOS}

Principio de la eliminacion .

5 minutos

Eliminación a los 15 minutos

$32 \%$

Eliminación de los 15 a los 30 minutos . . $23 \%$

Elimináción de los 30 a los 45 minutos . . $11 \%$

Eliminación de los 45

a $\operatorname{los} 60$ minutos.

Total eliminado una en hora

$71 \%$

Al estudiar detenidamente el cuadro anterior en el cual resumimos las 24 obscrviciones correspondientes a las enfermas de la primera mitad del embarazo, se observará:

$1^{\circ}$ Que el promedio encontrado para el tiempo de aparición del colorante en la orina, se encuentra dentro de los limites sijados como normales.

2. Que el promedio de eliminación total en una hora es ligeramente superior al que han fijado los autores americanos. Shaw y Young, como normal.

3. Que los promedios para los porcentajes de elininación en los diferentes cuartos de hora difieren de los que han encontrado los autores amerieanos, en el sentido de que en el primer cuarto de hora el poreen. taje estí disminuido, pero aumenta- do en los tres restantes, de tal manera que el porcentaje de elimina. ción total en una hora se encuentra normal.

En resumen, podemos decir que en la primera mitad del embarazo son muy pocas las modificaciones que experimenta la eliminación de la Sulfofenolptaleina.

En la segunda mitad del embara. zo, hemos observado que las modificaciones que experimenta la curva de eliminación de la Sulfofenolptaleina son mucho más marcadas. Los promedios que enco tramos sobre 70 enfermas fueron los siguientes:

\section{PROMEDIOS}

Principio de la eliminación 8 minutos

Eliminación a los 15 minutos . . . . $18 \%$

Eliminación de las 15
a $\operatorname{los} 30$ minutos . . $24 \%$

Eliminación de los 30

a $\operatorname{los} 45$ minutos. . $13 \%$

Eliminación de los 45

a $\operatorname{los} 60$ minutos . . $8 \%$

Total eliminado en una hora

$63 \%$

El estudio en conjunto del cuadro anterior nos ha permitido llegar a las conclusiones siguientes:

10 Que el ticmpo de aparición de la Sulfofenolptaleina en la orina esta retardado en la segunda mitad del embarazo y particularmente en los dos ditimos meses El maximum de retardo que hemos encontrado ha sido de 16 minutos 
$2^{\circ}$ Que el porcentaje de sustancia eliminado en el primer cuarto de hora está disminuido en proporción al retardo en el tiempo de aparición en la orina.

$3^{\circ}$ Que los porcentajes de eliminación para los tres cuartos de hora restantes están aumentados, compensando en esta forma la deficiente eliminación en el primero; esto nos explica el hecho de que los promedios para los porcentajes en cada uno de los cuartos de hora tiendan a ser más o menos iguales.

$4^{\circ}$ Que el parcentaje de eliminación total en una hora, a pesar de no habe: en algunos casos eliminación en l primer cuarto de hora, es en promedio de $63^{\prime}$. que no es muy bajo si se le compara con el fijado. como normal.

\section{Observaciones en la primera mifad del embarazo}

\begin{tabular}{|c|c|c|c|c|c|c|c|c|c|c|}
\hline \multirow{2}{*}{ Doser. } & \multirow{2}{*}{ Mlistario CHitrica } & \multirow{2}{*}{$\begin{array}{l}\text { Eded } \\
\text { Eñot }\end{array}$} & \multirow{2}{*}{ 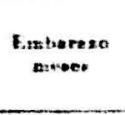 } & \multirow{2}{*}{ 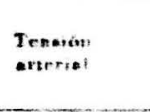 } & \multicolumn{2}{|c|}{ FH:BA DE } & \multicolumn{4}{|c|}{$\begin{array}{l}\text { LA SULFOERNOLPTALEINA } \\
\text { (Mintoe) }\end{array}$} \\
\hline & & & & & A presterit & is & 30 & is & of & SOTAE 9 \\
\hline 1 & $312 \quad A$ & 30 & 2 & $10 \ldots$ & $\vdots$ & 30 & 25 & 12 & 7 & 74 \\
\hline 2 & $378 \mathrm{M}$ & 32 & 2 & $12-6$ & 7 & 30 & 27 & 10 & 4 & 71 \\
\hline 3 & $511 \mathrm{M}$ & 30 & 2 & $10-6$ & 5 & 35 & 18 & 12 & 6 & 3 \\
\hline 4 & $603 A$ & 25 & 2 & $10.5-5$ & 4 & 35 & 20 & 10 & 3 & 68 \\
\hline 5 & $704 \mathrm{~A}$ & 19 & 2 & $115-7$ & 5 & 28 & 25 & 12 & 5 & 70 \\
\hline 6 & $532 \mathrm{~A}$ & 29 & 2 & $115-7$ & $\epsilon$ & 35 & 20 & 10 & 3 & 68 \\
\hline 7 & $605 \mathrm{M}$ & 28 & $21 / 2$ & $10.5-7$ & 5 & 10 & 30 & 20 & 6 & 56 \\
\hline 8 & $215 \mathrm{~A}$ & 23 & 3 & $12-7$ & 4 & 40 & 25 & 8 & 2 & 75 \\
\hline 2 & $374 \mathrm{M}$ & $2 !$ & 3 & $10 s-5$ & 8 & 15 & 27 & 20 & 10 & 72 \\
\hline 0 & $470 \mathrm{M}$ & 16 & 3 & $12-5$ & 7 & 35 & 17 & 15 & 6 & 73 \\
\hline 1 & $416 \mathrm{M}$ & 30 & 3 & $115-7$ & 4 & 40 & 20 & 7 & 5 & 72 \\
\hline 2 & 4218 & 16 & 3 & $11-7$ & 4 & 40 & 20 & 8 & 3 & $?:$ \\
\hline 3 & $780 \mathrm{~A}$ & 24 & 3 & $12-8$ & 10 & 10 & 35 & 15 & $b$ & 60 \\
\hline 4 & $662 \mathrm{M}$ & 19 & 3 & $9.5-6$ & 5 & 35 & 20 & 10 & 3 & 8 \\
\hline 5 & $414 \mathrm{~A}$ & 30 & $31 / 2$ & $115-5$ & $i_{i}$ & $3 !$ & 25 & 10 & 5 & 70 \\
\hline$\therefore$ & $326 \mathrm{M}$ & 23 & $3 / 2$ & $11-6$ & 4 & 40 & 15 & 10 & 6 & 71 \\
\hline 7 & $500 \mathrm{M}$ & 20 & $31 / 2$ & $10.5-5$ & 7 & 30 & 20 & 12 & 5 & 67 \\
\hline 8 & $661 M$ & 22 & $1 / 2$ & $11.5-5$ & 4 & 40 & 20 & 6 & 4 & 70 \\
\hline 9 & $740 \mathrm{~A}$ & 19 & $51 / 2$ & $11.5-7$ & 5 & 40 & 18 & 12 & 5 & 75 \\
\hline 2 & $388 \mathrm{~A}$ & 30 & 4 & $117-5$ & 5 & 38 & 20 & 12 & 4 & 74 \\
\hline 21 & $766 \quad A$ & 30 & $A$ & 10.55 & 9 & .8 & 35 & 12 & 3 & 5.8 \\
\hline $2 ?$ & $356 \quad A$ & 27 & 4. & $25-8$ & 4 & 35 & 20 & 10 & 3 & 58 \\
\hline 23 & $574 \mathrm{M}$ & 23 & $\because$ & 1258 & 3 & $\therefore 5$ & 15 & $Q$ & 4 & 72 \\
\hline .5 & $632 \mathrm{M}$ & 22 & $\therefore$ & $2: r$ & 4 & 40 & 20 & 10 & 5 & 75 \\
\hline
\end{tabular}




\section{Observaciones en la segunda miti f del embarazo}

\begin{tabular}{|c|c|c|c|c|c|c|c|c|c|c|}
\hline \multirow{2}{*}{$\begin{array}{l}\text { Obarer. } \\
\text { wecion }\end{array}$} & \multirow{2}{*}{$\begin{array}{l}1941 \\
\text { Bistoris Clinies }\end{array}$} & \multirow{2}{*}{$\begin{array}{l}\text { Edoet } \\
\text { - Aos: }\end{array}$} & \multirow{2}{*}{ Emitureno } & \multirow{2}{*}{$\begin{array}{l}\text { Teseoisn } \\
\text { ertertiat }\end{array}$} & \multicolumn{6}{|c|}{ 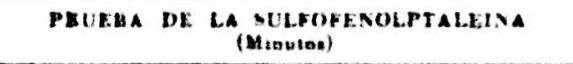 } \\
\hline & & & & & Aparición & 15 & 30 & 4 & 60 & Totai 9 \\
\hline 25 & $360 \quad A$ & 18 & 5 & $11.5-7$ & 8 & 20 & 15 & 15 & 15 & 65 \\
\hline 26 & $415 \mathrm{~A}$ & 20 & 5 & $12.7-5$ & 9 & 25 & 15 & 15 & 15 & 70 \\
\hline 27 & $624 \mathrm{M}$ & 19 & 5 & $105-5$ & 12 & 10 & 25 & 20 & 10 & 65 \\
\hline 28 & $706 \mathrm{M}$ & $2 !$ & 6 & $11-6$ & 6 & 40 & 17 & 10 & 4 & $7 !$ \\
\hline 29 & $692 \mathrm{M}$ & 19 & $61 / 2$ & $11.5-5$ & 7 & 15 & 25 & 17 & 10 & 67 \\
\hline 30 & $186 \AA$ & 22 & $61 / 2$ & $12.5-7$ & 5 & 27 & 20 & 17 & 10 & 74 \\
\hline 31 & $240 \mathrm{~A}$ & 23 & $61 / 2$ & $11-7$ & 7 & 30 & 17 & 12 & 8 & 67 \\
\hline 32 & $475 \mathrm{~A}$ & 22 & $6: / 2$ & $13.7-5$ & 6 & 20 & 27 & 15 & 10 & 72 \\
\hline 33 & $544 \mathrm{~A}$ & 19 & $61 / 2$ & $10.5-5$ & 5 & 25 & 15 & 12 & 10 & 62 \\
\hline 34 & $594 \quad A$ & 20 & $61 / 2$ & $12-8$ & 8 & 15 & 35 & 15 & 4 & 60 \\
\hline 35 & $602 \mathrm{~A}$ & 28 & 7 & $12.8-5$ & 7 & 10 & 15 & 30 & 15 & 70 \\
\hline 36 & $693 \mathrm{~A}$ & 24 & 7 & $11-7$ & 4 & 20 & 35 & 12 & 5 & 72 \\
\hline 37 & $635 \mathrm{~A}$ & 32 & 7 & $11.5-7$ & 7 & 27 & 20 & 12 & 8 & 67 \\
\hline 38 & $620 \mathrm{~A}$ & 18 & 7 & $12-c$ & 6 & 20 & 28 & 10 & 4 & $6 ?$ \\
\hline 39 & $604 \mathrm{~A}$ & 19 & 7 & $115-8$ & 9 & 15 & 20 & 15 & 10 & 60 \\
\hline 40 & $258 A$ & 23 & 7 & $125-?$ & 4 & 30 & 2.5 & 15 & 5 & 75 \\
\hline $4 !$ & $168 \mathrm{~A}$ & 17 & 7 & $12.6-5$ & 3 & 40 & 17 & 8 & 7 & 72 \\
\hline 42 & $474 \mathrm{~A}$ & 14 & 7 & $13.5-8$ & 0 & 25 & 30 & 15 & 6 & 76 \\
\hline 43 & $400 \mathrm{~A}$ & 23 & $71 / 2$ & $13-3$ & 7 & 35 & 18 & 10 & 5 & 68 \\
\hline 44 & $628 \mathrm{~A}$ & 28 & $71 / 2$ & $138-5$ & 10 & 10 & 30 & 20 & 5 & 65 \\
\hline 45 & $649 \mathrm{~A}$ & 23 & $7 / / 2$ & $10.5 \cdots-6$ & 11 & 5 & 30 & 25 & 10 & 70 \\
\hline 46 & $525 \mathrm{~A}$ & $2 c$ & $71 / 2$ & $12-8$ & 7 & 10 & 20 & 20 & 10 & $5 ?$ \\
\hline 17 & $693 \mathrm{~A}$ & 33 & $7 \%$ & $10.5-0$ & 6 & 35 & 20 & 15 & 5 & 75 \\
\hline 48 & $714 \mathrm{~A}$ & 34 & $7 ! / 2$ & $115 \%$ & 7 & 25 & 20 & 15 & 8 & 68 \\
\hline 49 & $548 \mathrm{M}$ & 22 & $7 ! / 2$ & $12-8$ & 15 & $\cdots$ & 40 & 15 & 12 & 67 \\
\hline 50 & $383 \mathrm{M}$ & 18 & $7 ! / 2$ & $10-6$ & 12 & 10 & 27 & 15 & 15 & 57 \\
\hline 5] & $340 \mathrm{M}$ & 18 & 8 & $11-5$ & 9 & 35 & 15 & $B$ & 4 & 62 \\
\hline 52 & $599 \mathrm{M}$ & 20 & 8 & $11.5--8$ & 11 & 5 & 35 & 20 & 10 & 70. \\
\hline 53 & $720 \mathrm{~A}$ & 22 & 8 & $12-7$ & 9 & 10 & 35 & 18 & 8 & $7 !$ \\
\hline 54 & $376 \quad A$ & 36 & 8 & $117-5$ & 9 & 27 & 15 & 15 & 12 & 69 \\
\hline 55 & $461 \mathrm{~A}$ & 26 & 8 & $12-7$ & 3 & 30 & 20 & 17 & 5 & 72 \\
\hline 55 & $527 \mathrm{~A}$ & 19 & 8 & 1285 & 9 & 25 & 20 & 12 & 5 & 62 \\
\hline 57 & $638 \mathrm{~A}$ & 20 & 8 & $11-7$ & 5 & 40 & 15 & 10 & 4 & 69 \\
\hline 58 & $636 \AA$ & 25 & 8 & $11.5-7$ & 3 & 15 & 35 & 12 & 6 & 60 \\
\hline 59 & 665 A & 22 & 8 & $11.5-7$ & 7 & is & 25 & 20 & 12 & 72 \\
\hline
\end{tabular}




\begin{tabular}{|c|c|c|c|c|c|c|c|c|c|c|}
\hline \multirow{2}{*}{$\begin{array}{l}\text { Shere. } \\
\text { recion }\end{array}$} & \multirow{2}{*}{$\begin{array}{l}1941 \\
\text { Biotoriat Clsices }\end{array}$} & \multirow{2}{*}{$\begin{array}{l}\text { Edad } \\
\text { SAos }\end{array}$} & \multirow{2}{*}{$\underset{\text { mesect }}{\text { Embaras }}$} & \multirow{2}{*}{$\begin{array}{l}\text { Tenaibe } \\
\text { erterial }\end{array}$} & \multicolumn{6}{|c|}{$\begin{array}{c}\text { PRUEBA DE LA SULFOFEROLPTALEINA } \\
\text { (Mintoe) }\end{array}$} \\
\hline & & & & & Apariciós & 15 & 30 & 45 & 60 & TOTAL \\
\hline 50 & $710 \AA$ & 23 & 8 & $11.5-7$ & 7 & 15 & 20 & 15 & 10 & 60 \\
\hline 51 & $727 \mathrm{~A}$ & 25 & 8 & $12-8$ & 5 & 22 & 30 & 12 & 8 & $? 2$ \\
\hline 2 & $140 \mathrm{M}$ & 20 & 8 & $12.5-7$ & 13 & $\mathrm{H}$ & 35 & 17 & 8 & 50 \\
\hline 3 & $195 \mathrm{M}$ & 20 & 8 & $11.5-7$ & 13 & $\mathrm{H}$ & 30 & 15 & 15 & 60 \\
\hline 4 & $591 \mathrm{~A}$ & 25 & 8 & $11.5-8$ & 4 & 15 & 25 & 15 & 10 & 65 \\
\hline 5 & $693 \mathrm{M}$ & 16 & 8 & $11.5-5$ & 11 & 10 & 25 & 15 & 6 & 56 \\
\hline 66 & $793 \mathrm{~A}$ & 17 & 8 & $138-5$ & 14 & $\mathrm{H}$ & 25 & 15 & 12 & 52 \\
\hline 67 & $268 \mathrm{M}$ & 21 & $81 / 2$ & $9.5-5$ & 16 & - & 32 & 12 & 10 & 54 \\
\hline 68 & 10) A & 24 & $84 / 2$ & $13-7$ & 12 & 5 & 28 & 15 & 8 & 56 \\
\hline$: 9$ & 347 A & 22 & $81 / 2$ & $11-7$ & 8 & 25 & 12 & 10 & 5 & 52 \\
\hline 70 & $625 \mathrm{~A}$ & 20 & $81 / 2$ & $12-8$ & 7 & 20 & 20 & 15 & 6 & 61 \\
\hline$\because$ & $798 \mathrm{~A}$ & 24 & $81 / 2$ & $13-8$ & 6 & 20 & 30 & 12 & 5 & 67 \\
\hline 2 & $454 \mathrm{M}$ & 30 & $81 / 2$ & $11.5-5$ & 8 & 15 & 25 & 15 & 6 & $6 !$ \\
\hline 3 & $333 \mathrm{M}$ & 28 & 9 & $12.5-7$ & 14 & $\mathrm{H}$ & 35 & 15 & 12 & 62 \\
\hline 4 & $756 \mathrm{~A}$ & 25 & 9 & $12.7--5$ & 5 & 20 & 35 & 12 & 6 & 73 \\
\hline 5 & $6: 2 \mathrm{~A}$ & 40 & 9 & $12.5-8$ & 8 & 32 & 15 & 12 & 5 & 64 \\
\hline 70 & $743 \mathrm{~A}$ & 22 & 9 & $9.5-6$ & 3 & 40 & 20 & 10 & 6 & 75 \\
\hline 77 & 1954 & 16 & 9 & $12-7$ & 10 & 5 & 30 & 18 & 7 & 50 \\
\hline 78 & $187 \mathrm{M}$ & 29 & 9 & $11.5-8$ & 14 & $\mathrm{H}$ & 30 & 12 & 12 & 54 \\
\hline 79 & $24 R$ & 35 & 9 & $11-7$ & 10 & 27 & 25 & 12 & 7 & 71 \\
\hline$\therefore 9$ & $398 \mathrm{~A}$ & 18 & 9 & $11.5-8$ & 12 & 4 & 35 & 12 & 10 & $6 !$ \\
\hline & $445 \mathrm{~A}$ & 20 & 9 & $11.5-7$ & 7 & 20 & 20 & 12 & 10 & 62 \\
\hline & $463 \mathrm{~A}$ & 25 & 9 & $10-5$ & 10 & 5 & 30 & 28 & 10 & 73 \\
\hline & $464 \quad \mathrm{~A}$ & 22 & 9 & $12.6-5$ & 8 & 10 & 20 & 15 & 15 & 60 \\
\hline & $442 \mathrm{M}$ & 18 & 9 & $116-5$ & 7 & 28 & 20 & 12 & 8 & 68 \\
\hline & 4431.1 & 19 & 9 & $12.5-8$ & 8 & 25 & 25 & 10 & $E$ & 65 \\
\hline & $590 \mathrm{~A}$ & 18 & 9 & $10.5-6$ & 12 & 5 & 30 & 15 & 8 & 58 \\
\hline 7 & $665 \mathrm{~A}$ & 32 & 9 & $127 \ldots 5$ & 7 & 40 & 25 & 7 & 5 & .77 \\
\hline$\therefore$ & $0.00 \mathrm{M}$ & 35 & $g$ & $10.5 \ldots 6$ & 6 & 38 & 20 & 12 & 5 & 75 \\
\hline 3 & $655 \mathrm{M}$ & 19 & 3 & $12--8$ & $\varepsilon$ & 15 & 35 & 12 & 6 & 68 \\
\hline 20 & $682 \mathrm{~A}$ & 22 & 9 & $11.7-5$ & 7 & 25 & is & 12 & 5 & 57 \\
\hline$\because:$ & $69 ! A$ & 20 & 9 & $12.5-8$ & 13 & $\mathrm{H}$ & 28 & 17 & 10 & 55 \\
\hline 9. & $697 \mathrm{M}$ & 16 & 9 & $125-8$ & 8 & 10 & 17 & 15 & 10 & $5 \%$ \\
\hline 92 & $579 \mathrm{~A}$ & 26 & 9 & $105-6$ & 4 & 38 & 15 & 10 & 5 & 63 \\
\hline 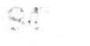 & $647 \mathrm{~A}$ & 20 & 9 & $138-5$ & 12 & 5 & 20 & 20 & 12 & 57 \\
\hline
\end{tabular}




\section{Observaciones en el puerperio}

\begin{tabular}{|c|c|c|c|c|c|c|c|c|c|c|}
\hline \multirow{2}{*}{$\begin{array}{l}\text { Ohenr: } \\
\text { veciás. }\end{array}$} & \multirow{2}{*}{$\begin{array}{l}2 y+1 \\
\text { Hiatoria Cllinie. }\end{array}$} & \multirow{2}{*}{$\begin{array}{l}\text { Fidend } \\
\text { ofices }\end{array}$} & & \multirow{2}{*}{$\begin{array}{l}\text { Tonaion } \\
\text { ertariel }\end{array}$} & \multicolumn{6}{|c|}{ PRCEBA DE LA SULYOEENOLPTALEINA } \\
\hline & & & & & Apericion & 15 & 30 & 45 & $\rightarrow 0$ & TOTALG \\
\hline 95 & $195 \mathrm{M}$ & 20 & & i $]-7$ & 6 & 30 & 28 & 10 & $A_{\hat{\gamma}}$ & 72 \\
\hline 95 & $3 n 1 M$ & 18 & & $12.7 \ldots 5$ & $A$ & 40 & 20 & 15 & 6 & 8! \\
\hline 57 & $268 \mathrm{M}$ & $2 i$ & . & $11.5-5$ & 7 & $3:$ & 20 & 12 & 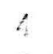 & $7 !$ \\
\hline 98 & $347 \mathrm{~A}$ & 22 & & $12.6-5$ & 6 & 32 & 18 & 10 & 5 & 65 \\
\hline 99 & $398 \quad A$ & 15 & & $10-5$ & 7 & 25 & 30 & 10 & 2 & 67 \\
\hline 100 & $445 \mathrm{~A}$ & 20 & & $11.5-6$ & 4 & 35 & 20 & 12 & 5 & 72 \\
\hline 101 & $464 A$ & 22 & & $12-8$ & 5 & 40 & 15 & 10 & 4 & 60 \\
\hline 102 & $463 A$ & 25 & & 105 & 5 & 35 & 25 & 10 & 5 & 75 \\
\hline 103 & $487 \quad A$ & 26 & & $115-7$ & 4 & 40 & 20 & 10 & 5 & 75 \\
\hline 104 & $333 \mathrm{k}$ & $2 \theta$ & & $13-8$ & 6 & 38 & 15 & 12 & 3 & 68 \\
\hline
\end{tabular}

De lo anteriormente expuest. se deduce claramente, que en el embarazo debe atrituirse mayor imprortancia a la eliminación total en una hora, que a la eliminación fraccionada por cuartos de hora. El hecho de haber encontrado en varias de nuestras observaciones un retardo en el tiempo de aparición en la orina $y$ un bajo porcentaje de eliminación o la falta de eliminación en el primer cuarto de hora, no debe traducirse por una insuficiencia renal. ya que los porcentajes de eliminación en los cuartos de hora restantes compensan esta deficiencia y la eliminación total en una hora se avecina a la normal.

Fste retardo en el tiempo de aparicion del colorante en la orina. $y$ la disminucion de un 10 a un $15 \%$ en el porcentaje total de eliminacion a una hora, que hemos eneontrato eri varias de nuestras enfermas aue tan sólo alcanzaron a eliminar 52 .
54,58 y $60 \%$, nos lo explicamos por las modificaciones de las vias urinarias provocadas por el embarazo.

Ha sido un hecho plenamente comprobado que durante el embarazo el ureter se dilata y sus contracciones peristalticas disminuyen en numero, intensidad y amplitud a medida que éste progresa, alcanzando su maximun de relajación hacia los dos últimos meses. Si tenemos en cuenta, que esta dilatación del ureter $y$ la atonia, más o menos completa de su musculatura, traen como consecuencia una retencicn de orina que puede variar entre 10 y $60 \mathrm{cc}$. una progresion mas lenta de la orina hacia la vejiga. nos explearemos fácilmente las modificaciones que hemos observado en la eliminacion do la Sulfofenolptaleina en la sefunda mitad del embarazo. En efecto La atonia del uriter y su dilatacion, con la consecuente retención te orina, nos explican suficientemente el re- 
tardo en el tiempo de aparición, la deficiente eliminación en el primer cuarto de hora, el aumento en los porcentajes de eliminación para los tres cuartos de hora restantes y la disminución de un 10 a un $15 \%$ en el porcentaje total eliminado en una hora.

Aceptada esta explicación satisfactoria y con el objeto de cerciorarnos de que ésta era su verdadera causa, investigamos en varias de uestras enfermas, en quienes habiamos practicado la prueba varias horas o varios dias antes del parto, si la Sulfofenolptaleina se había eliminado por la orina del feto, o si se ncontraba presente en el liquido amniótico y no habiéndonos sido posible comprobar, en ninguno de estos casos, su presencia en estos liquidos. desechamos la posibilidad de que cierta cantidad de la sustancia inyectada a la madre pasara a la circulacion fetal y se eliminara por la orina del feto.

Por demás está decir que esta hipótesis, es poco probable, ya que es logico pensar que si cierta cantidod de Sulfofenolptaleina se elimipor la orina del feto disminuendo asi los porcentajes de eliminaon, tal cosa debiera cuceder en totos los casos, y esto no lo hemos abrvado sino en un reducido numer. de enfermas.
Durante el puerperio, en 10 casos, er.contramos los promedios siguientes: Tiempo de aparición en la orina, 5 minutos; porcentaje de eliminación en el primer cuarto de hora, $35 \%$; segundo cuarto de hora, $21 \%$; tercer cuarto de hora, $11 \%$; cuarto cuarto de hora, $4 \%$; y total en una hora, $71 \%$. Como puede verse, estos promedios son muy similares a los que encontramos en la primera mitad del embarazo.

\section{Bibliografia.}

Cordaro G. The motor activity of the Ureter during Pregnancy Surgery, Gynecology and Obstetrics v. 66 . 1938.

Hundley J. M., Walton H. J. Physiological changes occurring in the urinary tract during Pregnaney. Am. J Obs. and Gynec. v. 30. 1935.

Hundley J. M. Siegel Isadore. Some Physiological and pathological observations on the urinary trac $d u$. ring Pregnancy. Surg. Gynec, and Obst. v. 66. 1938.

Irwing J. Strumph. Changes in the urinary tract during Pregnancy. Am. J. Obs. and Gynec, v. 26. 1933.

Ucrós Cuéllar Alfonso. La Sulfofenoptaleina en Crologia. Tesis de la Facultad de Medicina de Bogotá. 1936. 